\title{
Magnetic configuration effects on the Reynolds stress in the plasma edge
}

\author{
P. Manz $^{1}$, A. Stegmeir ${ }^{1}$, B. Schmid ${ }^{2}$, T. T. Ribeiro ${ }^{1}$, G. Birkenmeier ${ }^{3,1}$, \\ N. Fedorczak ${ }^{4}$, S. Garland ${ }^{2}$, K. Hallatschek ${ }^{1}$, M. Ramisch ${ }^{2}$, B. D. Scott ${ }^{1}$ \\ 1 Max-Planck-Institut für Plasmaphysik, 85748 Garching, Germany \\ 2 Institut für Grenzflächenverfahrenstechnik und Plasmatechnologie, Universität Stuttgart, 70569 \\ 3 Physik-Department E28, Technische Universität München, 85748 Garching, Germany \\ 4 CEA, IRFM, F-13108 Saint-Paul-Lez-Durance, France
}

(Dated: October 11, 2018)

\begin{abstract}
Breaking the poloidal symmetry of the magnetic shear induced tilt of turbulent structures, by either divertor X-point resistivity or limiter positions, can lead to a finite (residual) contribution to the flux-surface averaged radial-binormal Reynolds stress. This residual stress supports or works against the radial electric field at the plasma edge of a tokamak. The impact of divertor geometry on the poloidal pattern of the Reynolds stress is studied by flux-coordinate-independent fluid simulations. Clear modifications of the Reynolds stress are found due to the magnetic shear in the confined region. The impact of different poloidal limiter positions on the radial electric field and the Reynolds stress is studied by means of magnetic field aligned gyrofluid simulations. Only if the limiter is close to the outer midplane can its position have a substantial effect on the radial electric field.
\end{abstract}

PACS numbers:

\section{INTRODUCTION}

Understanding the influence of a complex magnetic field structure on plasma turbulence opens up new perspectives for the optimization of transport and significant improvements of confinement in magnetically confined fusion plasmas. One important example where the magnetic configuration influences the confinement is the position of the X-point. In the unfavorable configuration, where the ion $\nabla B$ drift is directed away from the $\mathrm{X}$-point, the power threshold of the $\mathrm{L}-\mathrm{H}$ transition is higher than in the favorable configuration, where the ion $\nabla B$ drift is directed towards the X-point [1-3]. The underlying mechanism so far is still unknown. Rotation in general is considered to be beneficial for magnetic confinement. Rotation can mitigate magnetohydrodynamical (MHD) macro-instabilities like the resistive wall mode [4]. Differential rotation is thought also to suppress MHD macro-instabilities like ballooning modes [5] in addition to the turbulence at micro-scales $[6,7]$. In contemporary experiments rotation is, to a large degree, externally provided by the external momentum input from neutral beam injection. Due to the large machine sizes of future reactor-grade devices like ITER or DEMO this may not be available. For this reason, rotation in the absence of externally applied torque, so-called intrinsic rotation, has recently been intensively studied $[8,9]$. Intrinsic rotation in the sense of Refs. $[8,9]$ refers to toroidal rotation mainly in the core region of the tokamak plasma. In the present study, self-generated (intrinsic) flows in the direction binormal to the magnetic field line and to the radial direction as well as flows in the poloidal direction are studied in the very edge of tokamak plasmas. Note that in a tokamak toroidal is not parallel and binormal is not poloidal. Toroidal and poloidal flows both have components in the parallel and binormal directions. Flows in the parallel and binormal directions both have components in the poloidal and toroidal directions. Therefore, with respect to broader research goals, the present study can be assigned to the topic of intrinsic rotation.

As mentioned above two of the main parameters determining confinement of magnetic fusion plasmas are magnetic and flow shear [7]. Flow shear, as well as magnetic shear, leads to a tilt of turbulent structures in the plane perpendicular to the magnetic field. The tilt of turbulent structures corresponds to a finite Reynolds stress $\left\langle\tilde{u}_{x} \tilde{u}_{y}\right\rangle$, where $\tilde{u}_{x, y}$ are the radial and binormal $E \times B$ velocity fluctuations and $\langle\cdot\rangle$ is an average specified in detail below. There are possible synergistic effects of magnetic and flow shear. Stronger magnetic shear leads to narrower layers of the zonal vorticity which is beneficial for confinement [10]. It is known that magnetic shear contributes to the $E \times B$ flow shearing rate $[11,12]$ and exhibits a contribution to the Reynolds stress [13]. This contribution can be due to a coupling to the radial-parallel Reynolds stress $\left\langle\tilde{u}_{x} \tilde{u}_{\|}\right\rangle$by the sheared slab approximation as in Ref. [13], however the tilt due to the magnetic shear can also directly influence the Reynolds stress as explained in the following.

Geometric modifications of the boundary conditions can influence the Reynolds stress [14]. In a single null configuration the $\mathrm{X}$-point breaks the poloidal symmetry of the magnetic shear induced tilt of turbulent structures, transmitting a finite contribution of the Reynolds stress to the flux-surface average. This residual Reynolds stress works either to reinforce or weaken the background flow shear. The sign of the residual stress depends on the magnetic configuration. In one configuration the flow shear is stronger and favorable and in the other the flow shear is weaker, which is unfavorable. Indeed changes of the energy transfer rate from the turbulence into the low frequency zonal flow (proportional to the Reynolds stress) are observed with changing poloidal position of 
the X-point [15]. The poloidal position of the limiter could have similar effects in principle.

For comparison with the dynamics in physical space, zonal flow modeling intrinsically requires flux surface averaged values of the Reynolds stress. However, not much is known about the spatial distribution of the Reynolds stress. In recent studies at the stellarator TJ-K [16], the Reynolds stress turned out to be strongly inhomogeneous on a flux surface. Thus, local measurements have to be interpreted with caution when comparing with theoretical predictions. This paper reports on the natural poloidal structure of the Reynolds stress as a result of purely magnetic shear in tokamak experiments. In addition, the impact of the poloidal limiter position and the presence of an X-point on the flux-surface averaged Reynolds stress is investigated by means of turbulence simulations.

This manuscript is organized as follows: The effect of magnetic shear on turbulent structures is compared to the effect of flow shear in the next (Sec. II). The development of residual Reynolds stress due to magnetic shear is also described in this section. The impact of divertor geometry on the poloidal pattern of the Reynolds stress is discussed and subsequently studied by means of flux-coordinate independent fluid simulations done with GRILLIX [17] in Sec. III. At present, GRILLIX does not feature finite ion temperatures. Therefore, a selfconsistent radial electric field is not present and the impact of the magnetic shear induced Reynolds stress on the radial electric cannot be studied with GRILLIX for the time being. Instead, we use the gyrofluid code GEMR $[18,19]$ to study this effect. GEMR includes a selfconsistent equilibrium, but does not include X-point geometry. The impact of different limiter positions on the radial electric field and the Reynolds stress will be discussed and subsequently studied in the circular-limited configuration using Tore Supra parameters, by means of field-aligned gyrofluid simulations in Sec. IV.

\section{MAGNETIC SHEAR INDUCED TILT OF TURBULENT STRUCTURES}

In the presence of magnetic shear $\hat{s}=\frac{\rho}{q_{s}} \frac{d q_{s}}{d \rho}$ (with radial coordinate $\rho$ and safety factor $q_{s}$ ) turbulent structures are progressively tilted as illustrated in Fig. 1. For positive magnetic shear $\hat{s}>0$ the safety factor $q_{s}$ is increasing with the radius. The field lines further outwards are less strongly twisted than further inwards. One can also understand this from the definition of the safety factor $q_{s}=N / M$, the ratio of toroidal $N$ to poloidal revolutions $M$, corresponding to the ratio of the toroidal $n$ to the poloidal mode number $m$. For $q_{s}=m / n$ increasing with radius $(\hat{s}>0)$, the poloidal mode number for a given toroidal mode number is increasing, hence the distance between the structures reduces with increasing radius. Whereas for the flux surfaces further inwards the poloidal mode is decreasing, hence the distance between

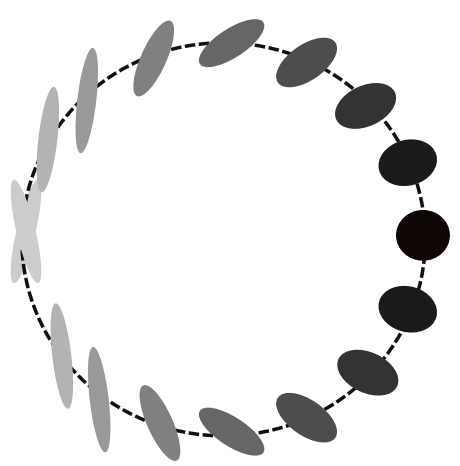

FIG. 1: Illustration of the tilt of turbulent structures by magnetic shear. It is shown how turbulent structures are tilted by the magnetic shear. The tilt induced by magnetic shear is up-down asymmetric.

the structures increases. In any case, magnetic shear leads to a poloidal tilt of magnetic flux tubes and associated turbulent structures. The tilt of the structures is equivalent to a finite Reynolds stress [20] which is given by $\left\langle\tilde{u}_{x} \tilde{u}_{y}\right\rangle$. In a plasma with circular cross-section the structures above and below the midplane are tilted in opposite directions (Fig. 1). Thus, the tilt induced by magnetic shear is up-down asymmetric. The geodesic curvature $\kappa_{g}$ in a tokamak with circular cross-section changes sign at the midplane and has a sinusoidal form with minimum and maximum at bottom and top. In Ref. [16] it has been found that the poloidal distribution of the Reynolds stress roughly follows the geodesic curvature. At smaller radii the flow is low and above the midplane the outer flow would point downwards (negative shear) whereas below the midplane the outer flow would point upwards (positive shear). In a limited plasma with circular cross section the confined region is poloidally symmetric, and therefore the magnetic shear should not affect the zonally averaged Reynolds stress. Therefore, only if the poloidal symmetry is broken, can magnetic shear lead to a residual contribution to the zonal flow drive.

Once a residual Reynolds stress is induced by the magnetic shear, it may act as a seed tilt, which can be selfamplified by the interaction of zonal flows and turbulence [14]. The Reynolds stress as the correlation between radial and perpendicular velocities $\left\langle\tilde{u}_{x} \tilde{u}_{y}\right\rangle \sim-k_{x} k_{y}\left|\tilde{\phi}^{2}\right|$ is proportional to the tilt which can be written in wavenumber space as $k_{x} k_{y}$. In an eikonal representation the radial wavenumber is subject to a change $k_{x}=-k_{y} \frac{\partial u_{y}}{\partial x} \tau_{c}$ if exposed to a sheared flow $u_{y}$ over the correlation time $\tau_{c}$. The magnetic shear $\hat{s}$ can lead to a tilt similar to the flow shear. Structures born at a particular position $\theta_{0}$ (for example the outboard midplane $\theta_{0}=0$ for curvatureinduced instabilities), which follow magnetic field lines are progressively tilted by the magnetic shear $k_{x}=k_{y} \theta \hat{s}$ $[14,21]$, where $\theta$ is the poloidal ballooning angle. The 
$\hat{s}$-induced Reynolds stress is given by $[14,21]$

$$
\Pi_{\hat{s}}(\theta)=-\hat{s} \theta\left\langle\tilde{u}_{x}^{2}(\theta)\right\rangle_{t},
$$

where $\langle\cdot\rangle_{t}$ denotes time average. The $\hat{s}$-residual Reynolds stress is given by the flux surface average $\left\langle\Pi_{\hat{s}}(\theta)\right\rangle_{\theta}$. In the case of a poloidally symmetric plasma, $\left\langle\Pi_{\hat{s}}\right\rangle_{\theta}$ vanishes. An imbalance between positive tilt and negative tilt can be provided by a poloidal truncation of the ballooning envelope, which can be provided by X-point resistivity [22] for diverted plasmas or the limiter position for limited plasmas $[14,21]$.

The derivation of Eq. (1) has been based on infiniteschanges of the phase due to the effect of magnetic shear. For this reason Eq. (1) is valid only for an infinitesimal distance $\Delta \theta=\theta-\theta_{0}$ from the original poloidal angle $\theta_{0}$, where the structure originates. This forces a linear dependence of Eq. (1) on the poloidal angle $\theta$. However, the entire behavior of the tilt is important, because what matters in the end is the flux-surface average of this quantity and only taking into account a narrow or even infinitesimal region around the low-field side midplane might not be a good approximation. Since the turbulence level is high at the midplane on the low-field side, one might be tempted to argue that only this region matters, however, one must not forget that the $\hat{s}$-induced Reynolds stress is small due to the linear $\theta$ dependence. Also Eq. (1) allows only for a periodic solution, important for the confined region, if $\tilde{u}_{x}^{2}(\theta)$ vanishes at the opposite ballooning angle to the angle where the structures are ently sheared structures coalesce coming from above and below the midplane. This leads to a cancellation of the $\hat{s}$-induced Reynolds stress. To take the coalescence into account, Eq. (1) can be modified according to imal changes of the radial wavenumber by infinitesimal generated $\tilde{u}_{x}^{2}\left(\theta_{0}+\pi\right)=0$. On the high-field side differ-

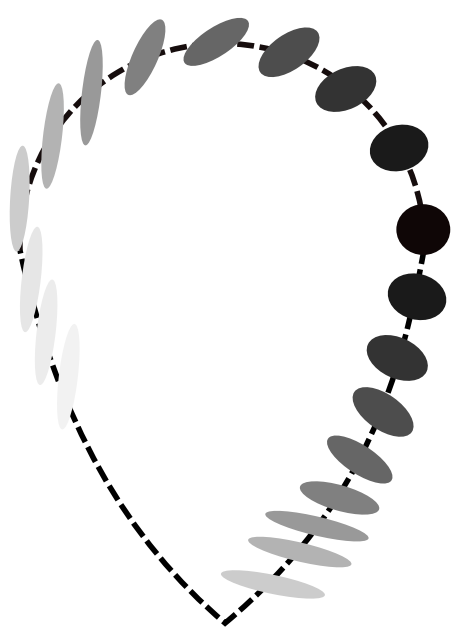

FIG. 2: Impact of an X-point on the tilt of turbulent structures. Similar figures can be found in Refs. [14, 21].

scans of the magnetic shear strength which are left for future studies. However, the sinusoidal pattern of the Reynolds stress is well imbedded in the dynamics of the plasma edge turbulence and is not isolated.

It should also be noted that the flow shear $V^{\prime}$ used in Refs. $[14,21]$ is subject to variation along the poloidal angle. As the potential is a flux-function it should be constant on a flux-surface, but the $E \times B$ flow and therefore also the shear depends on the magnetic field strength. Additionally flux expansion effects have to be taken into account.

\section{IMPACT OF THE X-POINT}

$\Pi_{\hat{s}}=u_{x}^{2}\left(\theta_{0}\right)\left(e^{-\frac{\left(\theta-\theta_{0}\right)^{2}}{\Delta \theta^{2}}} \hat{s}\left(\theta-\theta_{0}\right)-e^{-\frac{\left(2 \pi-\theta+\theta_{0}\right)^{2}}{\Delta \theta^{2}}} \hat{s}\left(2 \pi-\theta+\theta_{0}\right)\right)$ In the presence of an X-point the X-point resistivity

using $\tilde{u}_{x}^{2}(\theta)=\tilde{u}_{x}^{2}\left(\theta_{0}\right) e^{-\frac{\left(\theta-\theta_{0}\right)^{2}}{\Delta \theta^{2}}}$ as in Ref. [14]. This would provide a sinusoidal pattern of the Reynolds stress induced by tilt due to the magnetic shear as expected from basic considerations shown in Fig. 1.

It should be noted that a sinusoidal pattern of the Reynolds stress is part of the Pfirsch-Schlüter sideband balance [23]. A finite contribution of $\left\langle\tilde{u}_{x} \tilde{u}_{y} \sin \theta\right\rangle$ drives the flow sideband $\left\langle\tilde{u}_{y} \sin \theta\right\rangle$. This is in balance with the pressure sideband $\langle(p-W) \sin \theta\rangle$ (with $p$ the total pressure $p=p_{i}+p_{e}$ and the generalized potential built from electrostatic potential and ion pressure perturbations $W=\tilde{\phi}+\tilde{p}_{i}$, see Ref. [23] for more details) determines the Pfirsch-Schlüter balance $\langle(p-W) \sin \theta\rangle \sim\left\langle\tilde{J}_{\|} \cos \theta\right\rangle$. Therefore, the sinusoidal pattern of the Reynolds stress is coupled to the sinusoidal pattern in the pressure, which is part of the geodesic acoustic mode (GAM) oscillation. A stronger magnetic shear leads to stronger sidebands in $\left\langle\tilde{u}_{x} \tilde{u}_{y} \sin \theta\right\rangle$ and $\langle W \sin \theta\rangle$ which affects the overall sideband dynamics. A detailed investigation would require can act directly inside the confined region and therefore it can be expected that it has a much stronger effect on the Reynolds stress. The influence of the X-point on the tilt of turbulent structures by magnetic shear is illustrated in Fig. 2. Close to the outboard midplane the structures are expected to be tilted similarly to the case without X-point (Fig. 1). Approaching the X-point the structures become strongly elongated and thinned due to the strong magnetic shear close to the X-point. As very thin structures exhibit a high wavenumber these structures are strongly dissipated [22]. Therefore, the X-point directly truncates the ballooning envelope. This effect is not restricted to the scrape-off layer as in the limiter case described below. If the magnetic shear strongly varies with the ballooning angle, the magnetic shear $\hat{s}$ in Eq. (2) should be replaced by its local value $\frac{\rho}{q} \frac{\partial}{\partial \rho} \frac{B_{\varphi}(\theta)}{B_{\theta}(\theta)}$.

Approaches based on field-aligned coordinates suffer from coordinate singularities due to the X-points. Fluxcoordinate independent approaches offer the possibility to study these configurations. Such a flux-coordinate independent approach is done in the code GRILLIX 
[17]. An isothermal electrostatic drift-reduced Braginskii model [24] is simulated with different geometries. On the one hand GRILLIX takes into account a more realistic geometry. The effect of the X-point resistivity has already been demonstrated with GRILLIX as shown in Ref. [25]. On the other hand the simulations with GRILLIX do not take into account important physics necessary for the L-H transtion. One important part missing is the finite ion temperature, which is important for the radial electric field evolution $\left(E_{r} \sim\left(\nabla p_{i}\right) e n_{e}\right)[26]$, or electromagnetic effects. These effects are implemented in the code GEMR, which is used in the section IV, with the big disadvantage that GEMR does not allow study of an $\mathrm{X}$-point geometry.

As a first verification of our considerations we numerically investigate the effect of magnetic shear on individual structures. A typical field-aligned ballooninglike structure is considered in circular geometry without shear in Fig. 3a $\left(q_{0}=3, \hat{s}=0\right)$, with shear in Fig. 3c $\left(q_{0}=3, \hat{s}=0.75\right)$ and in diverted geometry in Fig. 3e. The structures are all poloidally symmetric Gaussians at the outboard midplane $\left(w_{\perp} / R_{0}=0.035\right.$ in the circular cases and $w_{\perp} / R_{0}=0.015$ in the diverted case) and field aligned with a Gaussian ballooning envelope along the parallel direction $\left(w_{\|} / R_{0} \approx 11 \approx \pi q_{0} R_{0}\right)$. The structures are tilted and distorted according to the respective magnetic geometry. Several toroidal positions are shown overlaid in the left column of Fig. 3. Assuming plasma parameters of the turbulence simulations presented later on $\left(\rho_{s} / R_{0}=1 \cdot 10^{-3}\right)$ the corresponding Reynolds stresses $\Pi=\left\langle\mathbf{e}_{\psi} \cdot \nabla u\left(\mathbf{e}_{\psi} \times \mathbf{e}_{\varphi}\right) \cdot \nabla u\right\rangle_{\varphi}$ of the individual structure $u$ are shown in the right column respectively (Figs. 3b,d,f). In the absence of magnetic shear the structure is not tilted (Fig. 3a) and is not associated with any Reynolds stress (Fig. 3b). Including magnetic shear, the structure is tilted (Fig. 3c) and the corresponding Reynolds stress shows the characteristic updown asymmetry (Fig. 3d), which confirms the picture in Fig. 1. In divertor geometry, including $\mathrm{X}$-points, the structure becomes strongly tilted and elongated at the upper high-field side and as well close to the lower X-point (Fig. 3e). Here we find the strongest contributions to the Reynolds stress from the individual structures (Fig. 3f). Keeping the structure and geometry fixed the Reynold stress scales like $\Pi \propto \rho_{s}^{-2}$, i.e. for the same structures the absolute values of the Reynolds stress at half $\rho_{s}$ would be four times larger. However, we want to note that instabilities and turbulence itself are also sensitive to $\rho_{s}$.

For comparison with our basic considerations (Fig. 1) and for comparisions with the GEMR simulations shown later, simulations in a circular cross-section are performed with GRILLIX. Since our aim is a qualitative comparison, no detailed matching of the exact parameters is needed and we used previously performed simulations. Simulations were done in a deuterium plasma with $R_{0}=1 \mathrm{~m}, B=0.5 \mathrm{~T}, n_{e}=2 \cdot 10^{18} \mathrm{~m}^{-3}, T_{e}=10 \mathrm{eV}$, $\tau_{e i}=4.4 \cdot 10^{-7} \mathrm{~s}$. More details can be found in chapters
5.1 and 5.3 of Ref. [17]. The radial-poloidal Reynolds stress $\left\langle\tilde{u}_{r} \tilde{u}_{\theta}\right\rangle_{\varphi, t}$ has been computed and is shown in Fig. 4. Here $\langle\cdot\rangle_{\varphi, t}$ denotes an average over the toroidal (axissymmetric) angle and time. As observed before in the GEMR simulations (Fig. 8), which were qualitatively consistent with our preliminary considerations (Fig. 1), an up-down asymmetry in the Reynolds stress is also observed here.

Figure 5 shows the radial-poloidal Reynolds stress $\left\langle\tilde{u}_{r} \tilde{u}_{\theta}\right\rangle_{\varphi, t}$ in divertor geometry. The up-down asymmetry is not as clear as in the circular cross-section. But on the high-field side some of this asymmetry appears to remain. Major contributions to the Reynolds stress can be found above the lower X-point and below the upper X-point. The enhanced magnetic shear in these regions impacts the tilt of the structures and thus the Reynolds stress. Very close to the lower X-point the Reynolds stress vanishes as the magnetic shear is too strong, and due to the strong thinning of the structures they are dissipated. This is what is meant by the $\mathrm{X}$-point resistivity. Close to the $\mathrm{X}$-point this $\mathrm{X}$-point resistivity acts to truncate the ballooning envelope. A bit further away the magnetic shear is not strong enough to dissipate the turbulent structures, but because the magnetic shear is very large the structures are very highly tilted leading to very strong contributions to the Reynolds stress. The basic structure of turbulence can in fact be traced back to the magnetic shear. The strongest contributions to the Reynolds stress in the case of turbulence (Fig. 5) can be found at the upper high-field side (positive Reynolds stress) and close to the lower X-point (negative Reynolds stress), which also show up for individual structures (Fig. 3f). Thus, to first order the poloidal variation of the Reynolds stress can be understood as the effect of magnetic shear on individual structures.

On the low-field side the Reynolds stress does not show a strong up-down asymmetry but instead is organized in bands with radially alternating signs. This is expected from a zonal flow staircase-like structure. In the case of the GEMR simulations the background shear $\left(E_{r} \sim\right.$ $\left.\nabla p_{i} /(e n)\right)$ may lock the zonal flow pattern, whereas in the present GRILLIX simulations the zonal flow is more free.

\section{IMPACT OF THE LIMITER POSITION}

In the scrape-off layer (SOL) region the position of the limiter may induce a truncation of the ballooning envelope and can lead to a finite contribution to the zonally averaged Reynolds stress. Three examples are shown in Fig. 6. If the limiter is placed at the bottom (Fig. 6a) or the top (Fig. 6b) the fluctuation level directly behind the limiter is reduced, which leads to an up-down asymmetry. Besides the point directly at the low-field side (LFS) midplane where the asymmetry vanishes by definition, the asymmetry increases the closer the limiter is located to the LFS midplane (Fig. 6c). Direct impact of the lim- 


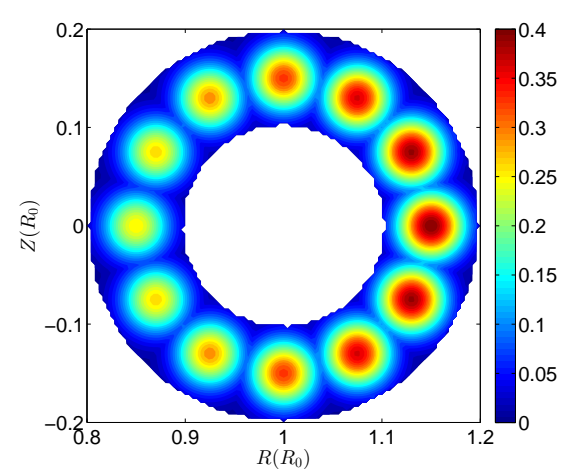

(a)

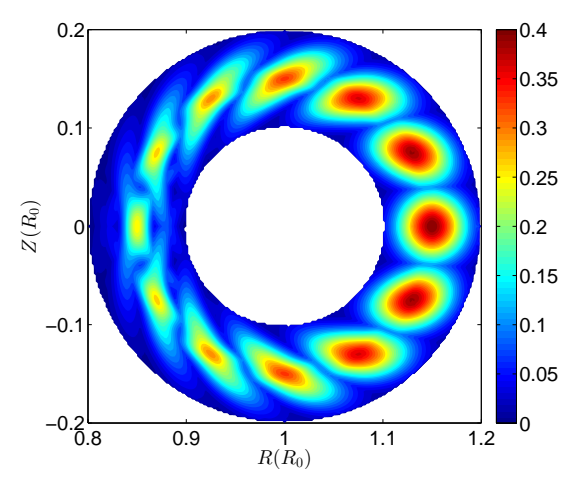

(c)

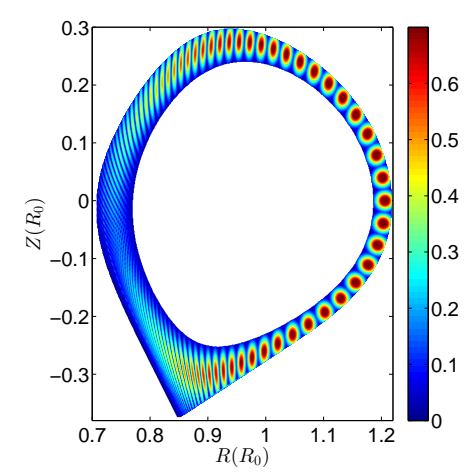

(e)

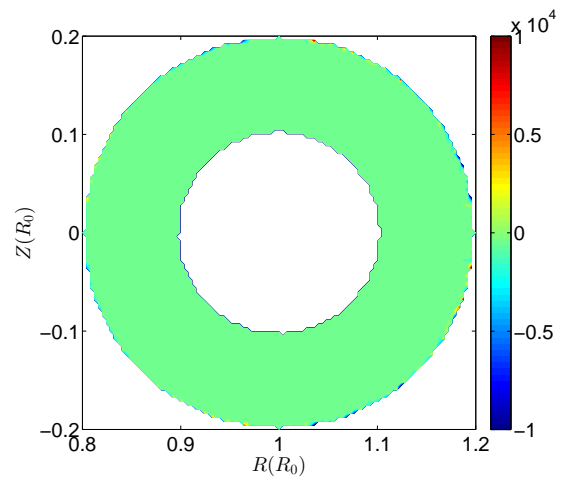

(b)

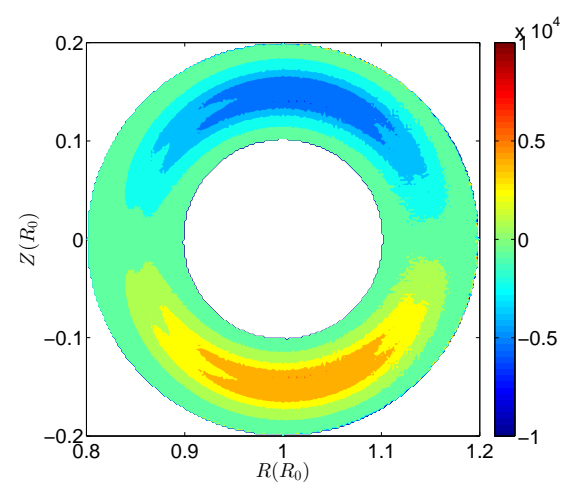

(d)

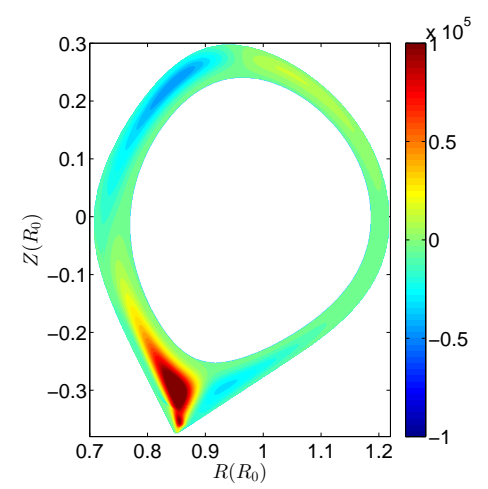

(f)

FIG. 3: GRILLIX simulations: The left hand side shows poloidal cross-sections of an individual structure starting at the outboard midplane and summed over several toroidal positions (a,c,e). Assuming the same plasma parameters as in the turbulence simulations presented later on $\left(\rho_{s} / R_{0}=1 \cdot 10^{-3}\right)$, and identifying the structure with normalized potential $e \phi / T_{e}$, the corresponding Reynolds stress is shown on the right hand side $(\mathrm{b}, \mathrm{d}, \mathrm{f})$ in units of $\left[\mathrm{m}^{2} / \mathrm{s}^{2}\right]$. Both are shown in the case of a circular cross-section without magnetic shear $(a, b)$, with magnetic shear $(c, d)$ and in the case of a diverted plasma $(\mathrm{e}, \mathrm{f})$.

iter is restricted to the SOL region. However, a finite contribution to the zonally averaged Reynolds stress at the LCFS, at the boundary of the confined region, can impact the edge region, which may be nonlinearly selfamplified. This can only be found out via simulations.

The influence of the limiter position has been investigated with the help of simulations carried out with the three-dimensional gyrofluid electromagnetic turbulence model GEMR $[18,19]$. GEMR is a six-field model and simulates the densities, parallel velocities, parallel and perpendicular temperatures and parallel and perpendicular parallel heat fluxes for ions and electrons respectively. These are the dependent variables. Potential perturbations with finite Larmor radius corrections are 


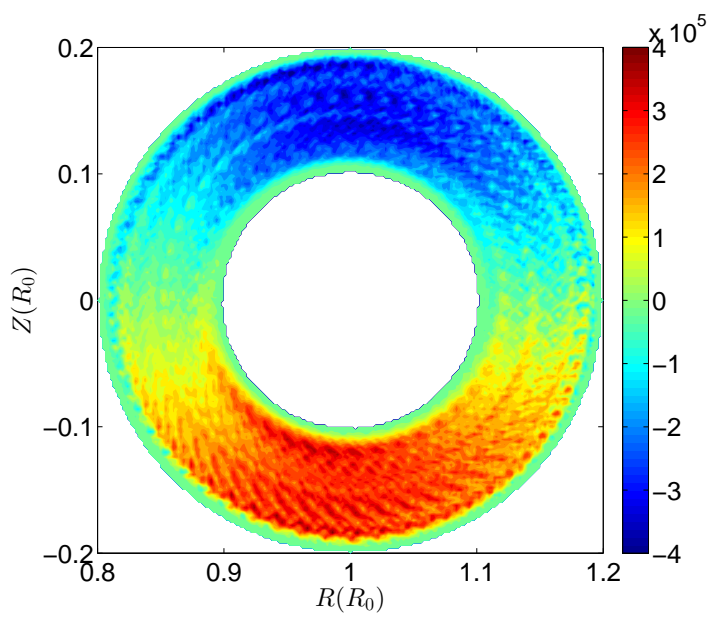

FIG. 4: Reynolds stress $\left\langle\tilde{u}_{r} \tilde{u}_{\theta}\right\rangle_{\varphi, t}$ in $\mathrm{m}^{2} / \mathrm{s}^{2}$ in the poloidal plane in a circular cross-section simulated with GRILLIX including finite magnetic shear.

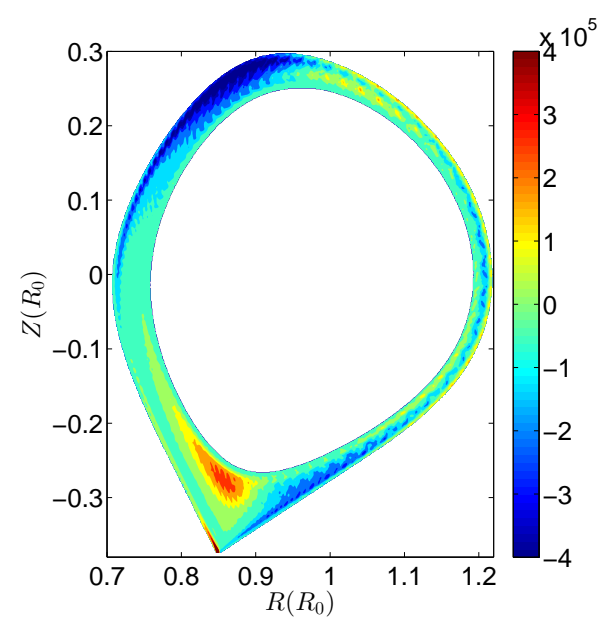

FIG. 5: Reynolds stress $\left\langle\tilde{u}_{r} \tilde{u}_{\theta}\right\rangle_{\varphi, t}$ in $\mathrm{m}^{2} / \mathrm{s}^{2}$ in the poloidal plane in a diverted configuration with $\mathrm{X}$-point simulated with GRILLIX.
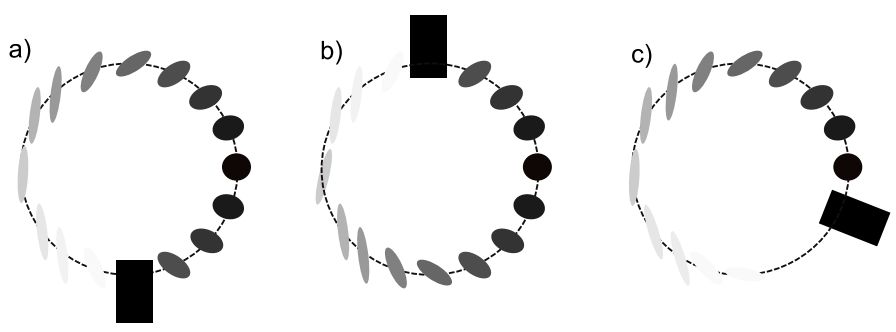

FIG. 6: Impact of the limiter position on the tilt of turbulent structures. deduced from quasineutrality. A circular plasma crosssection with toroidal axisymmetry is implemented. The coordinate system is aligned with a self consistent time varying equilibrium magnetic field. The gradients evolve freely, as required by the strength of fluctuating dynamics in the plasma edge region. The profiles are also included in the dependent variables in the polarization equation, and so GEMR is a global model. The background radial electric field evolves self-consistently. Details on the self-consistent treatment of the profiles and MHD equilibrium can be found in Ref. [18]. In summary, GEMR enables self-consistent simulation of an equilibrium (providing the radial electric field $E_{r}$ ) together with the turbulence (providing the Reynolds stress).

The coordinate system $(x, y, s)$ is magnetic field aligned, with $x$ in the radial, $y$ in the binormal and $s$ in the parallel direction relative to the magnetic field vector. The set of grid points with constant parallel coordinate represents the drift plane, which is perpendicular to the magnetic field. The open field lines corresponding to the SOL are implemented by the boundary conditions via a perturbed Debye sheath current [27]. The actual boundary conditions used in the simulation, which include the temperature dynamics, can be found in Ref. [28]. The deviation from the preset background values has to be small as required by consistency. The input parameters are $\rho^{*}=\delta=\rho_{s} / a$, plasma beta $\beta$, and normalized collisionality $\nu=a \nu_{e} / c_{s}$. Here $\rho_{s}=\sqrt{T_{e} m_{i}} / e B$ is the hybrid Larmor radius, $c_{s}=\sqrt{T_{e} / m_{i}}$ is the ion sound speed, $a$ is the minor radius, $T_{e}$ is a reference electron temperature, $B$ is the magnetic field strength and $\nu_{e}$ is the inverse Braginskii collision time.

As GEMR is limited to circular plasma poloidal cross sections a comparison with experiments in such a geometry is desirable. In Ref. [21] the model of the $\hat{s}$-induced residual stress [14] has been compared to experiments in Tore Supra. Tore Supra $(R=2.4 \mathrm{~m}, a=0.70 \mathrm{~m})$ is usually operated at high magnetic fields $(B=3.5$ $\mathrm{T})$ with roughly circular plasma poloidal cross section. Fortunately, the geometry of GEMR is suitable for Tore Supra modeling. The experimental parameters at the LCFS (the reference flux surface) are taken from Ref. $[14,21]$ to be $n_{e}=4 \cdot 10^{18} \mathrm{~m}^{-3}, T_{e}=T_{i}=40 \mathrm{eV}$ and $L_{n}=L_{T e}=L_{T i}=3.5 \mathrm{~cm}$. These translate to the following GEMR input parameters: $\delta=4.27 \cdot 10^{-4}$, $\beta=2.79 \cdot 10^{-6}, \nu=9.26$. The simulations have been done on a $256 \times 512 \times 16$ grid. The radial resolution is $\rho_{s}$, the binormal resolution is about $2 \rho_{s}$ which is marginal but at least $k_{y} \rho_{s}=1$ where the strongest interaction between shear flows and turbulence takes place [29] is resolved. At the LCFS the safety factor is $q_{s}=3.5$ with a magnetic shear of $\hat{s}=1.4$. In the experiments described in Ref. [21] the limiter positions were $\theta_{X}= \pm 35^{\circ}$, whereas $\theta_{X}= \pm 33.75^{\circ}$ have been simulated.

In the lower limiter case, $\theta_{X}=-33.75^{\circ}$, (Fig. 7a) the radial electric field is deeper at the seperatrix than in the upper limiter case, $\theta_{X}=+33.75^{\circ}$ (Fig. 7b). Therefore, in the GEMR simulations the lower limiter case shows 

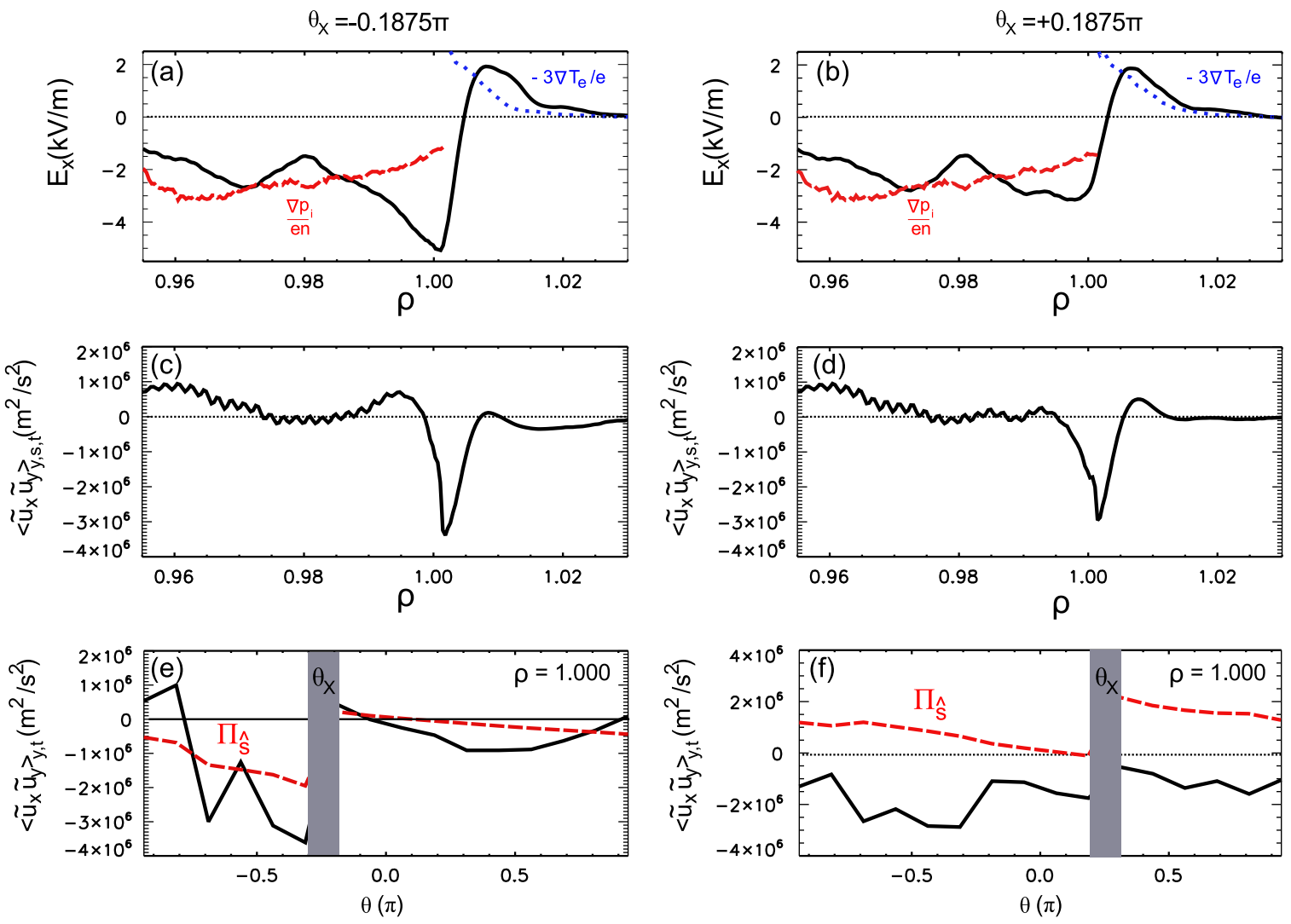

FIG. 7: GEMR simulations of Tore Supra discharges. Radial electric field (a,b), radial profile of the zonally averaged Reynolds stress (solid line) (c,d), poloidally resolved contribution to the Reynolds stress (solid black line) and the $\hat{s}$-residual Reynolds stress contribution (red dashed line) at the separatrix $\rho=1.00$ (e,f) for the lower $\theta_{X}=-33.75^{\circ}(\mathrm{a}, \mathrm{c}, \mathrm{e})$ and upper limiter case $\theta_{X}=+33.75^{\circ}(\mathrm{b}, \mathrm{d}, \mathrm{f})$.

better confinement properties for the favorable configuration. The radial electric fields are similar to the experimental values in Tore Supra [21]. The GEMR simulations found minima in the radial electric field at around 6 and $3 \mathrm{kV} / \mathrm{m}$ in the lower and upper limiter case, respectively. In the Tore Supra experiment the minima in the radial electric field for the lower and upper limiter case are at 7 and $4 \mathrm{kV} / \mathrm{m}$ [21]. Here, the ion diamagnetic velocity is defined negative for easier comparison with Refs. [14, 21]. Therefore, the shear across the LCFS is negative. Turbulence structures tilted in the direction of this shear exhibit a negative zonally averaged Reynolds stress as observed in Figs. 7c and d. When the zonally averaged Reynolds stress drive $-\partial_{x}\left\langle\tilde{u}_{x} \tilde{u}_{y}\right\rangle_{y, s, t}$ is positive (Figs. 7c and $\mathrm{d}$ ) this leads to an acceleration in electron diamagnetic direction (Figs. 7a and b). The zonally averaged Reynolds stress in the lower limiter case (Fig. 7c) is of similar order of magnitude to, but steeper than, than the upper limiter case (Fig. 7d). Therefore both the Reynolds stress drive $-\partial_{x}\left\langle\tilde{u}_{x} \tilde{u}_{y}\right\rangle_{y, s, t}$ as well as the turbulence suppression $\left\langle\tilde{u}_{x} \tilde{u}_{y}\right\rangle_{y, s, t} \partial_{x}\left\langle u_{y}\right\rangle_{y, s, t}$ are stronger in the former case. Here $\langle\cdot\rangle_{a}$ are the corresponding zonal (binormal and parallel) and time averages, with $a \in\{y, s, t\}$. Deeper into the confined region $(\rho<0.99)$ the Reynolds stress shows marginal impact on the limiter position (Figs. 7c and $\mathrm{d}$ and 8). The Reynolds stress is small at the outer midplane $(\theta=0)$, increases towards negative $\theta$ and decreases towards positive $\theta$. Both contributions largely cancel out in the zonally averaged value (Fig. 7c and d). As expected by our preliminary consideration in the case of dominant impact of the magnetic shear (Fig. 1), the Reynolds stress mainly shows an up-down asymmetric $m=1$ structure in the confined region, as shown in Fig. 8. This is not affected by the limiter position. Therefore, the impact of the magnetic shear on the structure tilt is stronger than the impact of the flow shear, and the limiters cannot influence the plasma far into the confined region.

Next, the SOL dynamics are investigated which is somewhat more complicated. Structures are born around the outboard midplane $\theta_{0}=0$, where the tilt and the Reynolds stress is low. As structures follow the magnetic field lines they get progressively tilted by the magnetic shear $k_{x}=k_{y} \theta \hat{s}$. Structures following the negative $\theta$ direction become tilted in agreement with the magnetic shear leading to a positive Reynolds stress (Fig. 8). Structures following positive $\theta$ exhibit a negative Reynolds stress (Fig. 8). In the limiter shadow 


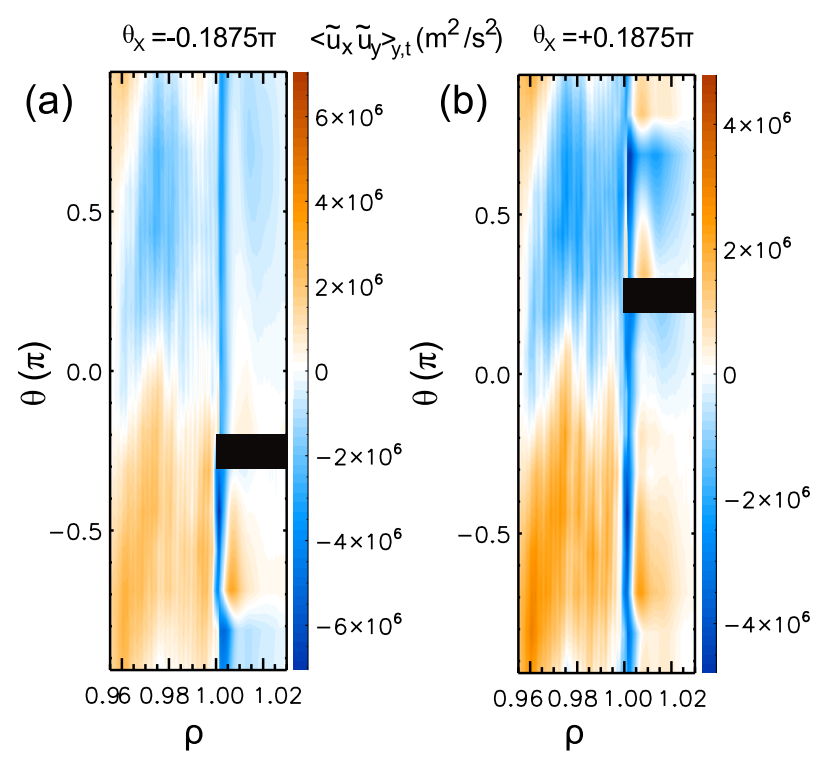

FIG. 8: GEMR simulations of Tore Supra discharges.

Poloidally and radially resolved Reynolds stress contribution $\left\langle\tilde{u}_{x} \tilde{u}_{y}\right\rangle_{y, t}$ for the upper limiter case $\theta_{X}=+33.75^{\circ}$ (a) and the lower limiter case $\theta_{X}=-33.75^{\circ}(\mathrm{b})$.

the Reynolds stress distribution can be understood in a similar way. Below the limiter in Fig. 8a and above the limiter in Fig. 8b plasma comes not directly from the outboard midplane, but radially penetrates into this region. Structures very close to the limiter do not propagate a large distance along the field lines and do not get tilted much, therefore they do not produce significant Reynolds stress. The structures penetrating the limiter shadow region with some distance to the limiter are again tilted by magnetic shear leading to a positive (negative) Reynolds stress for lower (higher) $\theta$ values. At some $\theta$ structures originating at the outboard midplane region coalesce with structures penetrating the limiter shadow region. This is the reason for the second point of vanishing Reynolds stress at around $-\theta=0.75 \pi$ and $\theta=0.75 \pi$ in Fig. 8a and $\mathrm{b}$, respectively. Directly at the separatrix the situation is similar but due to the strong negative shear the Reynolds stress exhibits a strong negative baseline value, in particular in the region $[-\pi, 0]$ from the outboard midplane towards the bottom of the inboard midplane.

Figure 9a shows the impact of the poloidal limiter position on the minimum of the radial electric field. The minimum of the radial electric field changes by a factor of two from about $6 \mathrm{kV} / \mathrm{m}$ for the limiter at the midplanes to $3 \mathrm{kV} / \mathrm{m}$ for limiters at the bottom or top. The impact of the limiter position is stronger if the limiter is closer to the midplane. A similar variation is observed for the minimum of the Reynolds stress (Fig. 9b), which changes barely by a factor of two, with the strongest Reynolds stress obtained with the limiters at the midplanes. To estimate the $\hat{s}$ induced residual Reynolds stress an initial
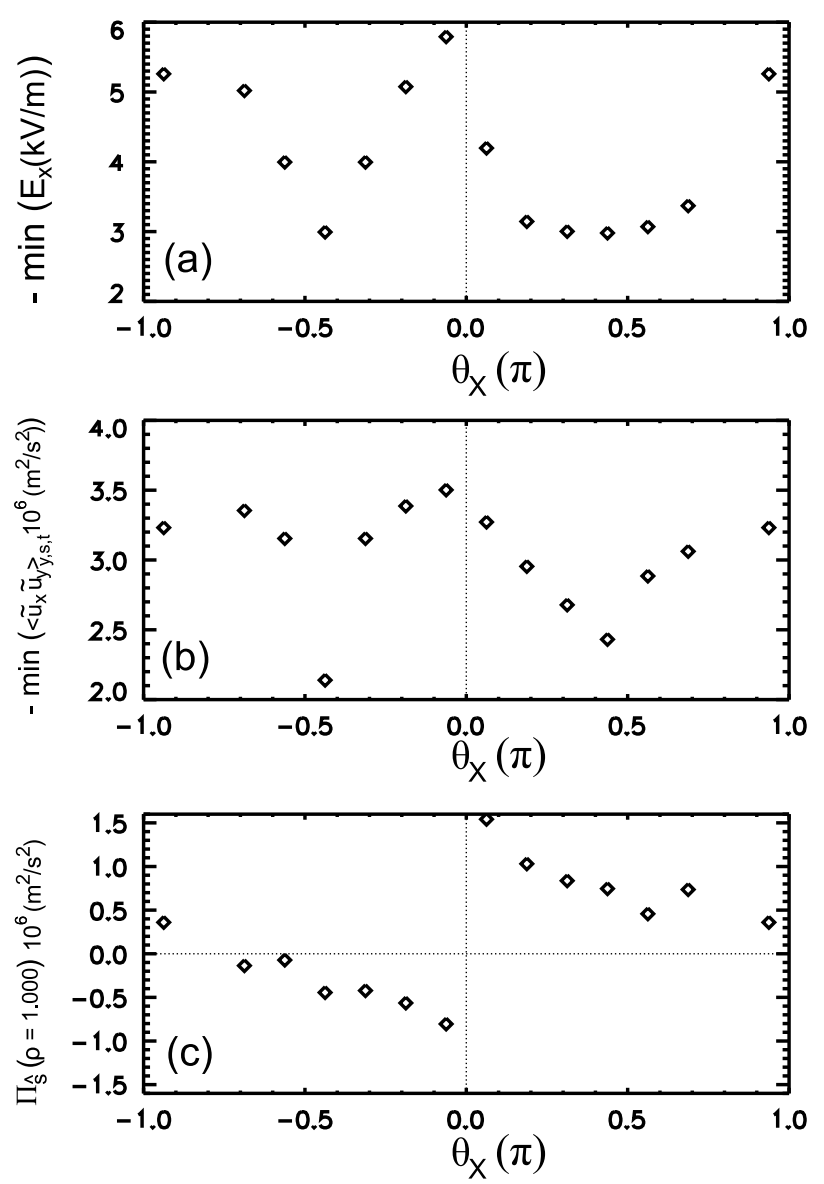

FIG. 9: GEMR simulations of Tore Supra discharges. Minimum of the zonally averaged radial electric field (a) and zonal averaged Reynolds stress (b) and $\hat{s}$-residual Reynolds stress (c).

position $\theta_{0}$ has to be set. This has been chosen to be the poloidal position of maximum amplitude in the potential fluctuations. According to this calculated $\hat{s}$-induced residual Reynolds stress is shown in Fig. 9c. Qualitatively it shows a modulation with respect to the limiter position as predicted (Fig.4a of Ref. [21]). As the turbulence exhibits its maximum in amplitude around the outboard midplane the variation of the limiter position has the highest impact if the limiter is placed in this region (Fig. 6). If the limiter is far away from the outboard midplane the main part of the turbulence will be symmetrically tilted and no effect of the $\hat{s}$-residual stress can be expected.

\section{SUMMARY}

The poloidal distribution of the Reynolds stress has been investigated by means of field-aligned gyrofluid simulations carried out with the GEMR code and with fluxcoordinate independent fluid simulations carried out with 
the GRILLIX code. In the confined region of a plasma with circular plasma cross section the poloidally resolved Reynolds stress $\left\langle\tilde{u}_{x} \tilde{u}_{y}\right\rangle_{y, t}(\theta)$ is mainly up-down asymmetric (Figs. 8 and 4 ), which is in agreement with the structures being tilted by magnetic shear (Fig. 1). In the zonally average the up-down asymmetric contributions to the Reynolds stress cancel and the remaining zonal averaged Reynolds stress is rather small.

The impact of X-point resistivity [14] (Fig. 2) on the poloidal distribution of the Reynolds stress has been studied by means of flux-coordinate-independent fluid simulations carried out with GRILLIX. Indeed, some tokamaks without divertors have never achieved H-mode plasmas and the power threshold for limited plasmas is higher than that for diverted plasmas. Diverted plasmas have also been studied by means of flux-coordinateindependent fluid simulations carried out with GRILLIX. In the case of a circular cross-section an up-down asymmetric Reynolds stress is also observed (Fig. 4). For diverted geometry, the Reynolds stress shows a complicated poloidal pattern (Fig. 5), which can be traced back to the effects of magnetic shear on individual structures starting at the outboard midplane (Fig. 3e and f).

The impact of the poloidal limiter position on the Reynolds stress has been studied with gyrofluid simulations carried out using the GEMR code. The GEMR simulations reproduce the experimental situation in Tore Supra quite well [21]. By changing the poloidal limiters by $\theta_{X}= \pm 33.75^{\circ}$ with respect to the outer midplane angle the minimum of the radial electric field changes by a factor of two (Fig. 7a and b). There is no strong impact on the zonally averaged Reynolds stress and radial electric field unless the limiters are very close to the outer midplane (Figs. 6 and 9). For the case of limiters at the top or bottom, which would correspond to lower or upper single null configuration, the impact of the limiter position does not show a significant difference. In general, the impact of the limiters on the confined region is limited to the very edge (up to $\rho=0.99$ at most). A strong nonlinear feedback of the modifications at the boundary was absent in the present simulations although the simulation could provide this possibility in principle. This shows the impact of the poloidal position of the limiter on the confinement cannot be very strong.

The simulations carried out here reveal the strong poloidal asymmetry of the Reynolds stress. Hence, measurements at one poloidal positions should not be taken as representative of the zonal average. For this reason, it is not surprising that experiments on different devices, with diagnostics at different positions, find differences in sign and strength of the Reynolds stress. In some investigations of the impact of the Reynolds stress with respect to the $\mathrm{L}-\mathrm{H}$ transition it was found that the Reynolds stress is strong enough [30-35] to trigger the L-H transition, but in others it was not strong enough $[36,37]$ (even with a different sign in Ref. [37]). To point this out explicitly, it is possible to both over and underestimate the flux-surface average Reynolds stress by taking a local measurement only. The Reynolds stress have been experimentally found to have the opposite sign at two different poloidal locations [38]. From the present study it can be concluded that a better understanding of the spatial distribution of the Reynolds stress is necessary for a proper interpretation of local measurements involving simulations with realistic field geometry.

\section{Acknowledgements}

This work has been carried out within the framework of the EUROfusion Consortium and has received funding from the Euratom research and training programme 2014-2018 under grant agreement No 633053. The support from the EUROfusion Researcher Fellowship programme under grant number AWP16-ERGMPG/Stegmeir is gratefully acknowledged. The views and opinions expressed herein do not necessarily reflect those of the European Commission.
[1] ASDEX team, Nucl. Fusion 29, 1959 (1989).

[2] J. A. Snipes R. S. Granetz, M. Greenwald, O. J. W F. Kardaun, A. Kus, F. Ryther, U. Stroth, J. Kollermeyer, S. J. Fielding, M. Valovic, Nucl. Fusion 36, 1217 (1996).

[3] A. E. Hubbard R. L. Boivin, J. F. Drake, M. Greenwald, Y. In, J. H. Irby, B. N. Rogers, J. A. Snipes, Plasma Phys. \& Controlled Fusion 40, 689 (1998).

[4] D. J. Ward and A. Bonderson, Phys. Plasmas 2, 1570 (1995)

[5] R. L. Miller, F. L. Waelbroeck, A. B. Hassam, and R. E. Waltz, Phys. Plasmas 2, 3676 (1995).

[6] H. Biglari, P. H. Diamond, and P. W. Terry, Phys. Fluids B: Plasma Phys. 2, 1 (1990).

[7] K. H. Burrell, Phys. Plasmas 4, 1499 (1997).

[8] J. Rice, A. Ince-Cushman, J. deGrassie, L.-G. Eriksson,
Y. Sakamoto, A. Scarabosio, A. Bortolon, K. Burrell, B. Duval, C. Fenzi-Bonizec, M. Greenwald, R. Groebner, G. Hoang, Y. Koide, E. Marmar, A. Pochelon, and Y. Podpaly, Nucl. Fusion 47, 1618 (2007).

[9] Y. Camenen, C. Angioni, A. Bortolon, B. P. Duval, E. Fable, W. A. Hornsby, R. M. McDermott, D. H. Na, Y.-S. Na, A. G. Peeters, and J. E. Rice, Plasma Physics and Controlled Fusion 59, 034001 (2017).

[10] A. Kendl and B. D. Scott, Phys. Rev. Lett. 90, 035006 (2003).

[11] T. S. Hahm and K. H. Burrell, Phys. Fluids 2, 1648 (1995).

[12] T. S. Hahm, D. H. Na, J. W. Lee, J. W. Park, Y. S. Na, S. S. Kim, W. H. Ko, P. H. Diamond, H. Jhang, and Y. M. Jeon, Nucl. Fusion 53, 093005 (2013).

[13] H. Sugama and W. Horton, Phys. Plasmas 1, 345 (1994). 
[14] N. Fedorczak, P. H. Diamond, G. R. Tynan, and P. Manz, Nucl. Fusion 52, 103013 (2012).

[15] I. Cziegler, A. E. Hubbard, J. W. Hughes, J. L. Terry, and G. R. Tynan, Phys. Rev. Lett. 118, 105003 (2017).

[16] B. Schmid, P. Manz, M. Ramsich, and U. Stroth, New. J. Physics 19, 055003 (2017).

[17] A. Stegmeir, D. Coster, A. Ross, O. Maj, K. Lackner, and E. Poli, Plasma Phys. \& Controlled Fusion 60, 035005 (2018).

[18] B. D. Scott, Contrib. Plasma Phys. 46, 714 (2006).

[19] A. Kendl, B. D. Scott, and T. T. Ribeiro, Phys. Plasmas 17, 072302 (2010).

[20] A. Liberzon, B. Lüthi, M. Guala, W. Kinzelbach, and A. Tsinober, Phys. Fluids 17, 095110 (2005).

[21] N. Fedorczak, P. Ghenrih, P. Hennequin, G. R. Tynan, P. H. Diamond, and P. Manz, Plasma Phys. \& Controlled Fusion 55, 124024 (2013).

[22] D. Farina, R. Pozzoli, and D. D. Ryutov, Nucl. Fusion 9, 1315 (1993).

[23] B. D. Scott, New J. Phys. 7, 92 (2005).

[24] A. Zeiler, J. F. Drake, and B. Rogers, Phys. Plasmas 4, 2134 (1997).

[25] A. Stegmeir, D. Coster, O. Maj, A. Ross, and K. Lackner, Proc. of the $26^{t h}$ IAEA Fusion Energy Conference, Kyoto, Japan , TH/P6-6 (2016).

[26] M. Cavedon, T. Pütterich, E. Viezzer, G. Birkenmeier, T. Happel, F. M. Laggner, P. Manz, F. Ryter, U. Stroth, and Asdex Upgrade Team, Nucl. Fusion 57, 014002 (2017).

[27] T. T. Ribeiro and B. Scott, Plasma Phys. \& Controlled Fusion 47, 1657 (2005).

[28] T. T. Ribeiro and B. Scott, Plasma Phys. \& Controlled
Fusion 50, 055007 (2008).

[29] P. Manz, M. Ramisch, and U. Stroth, Phys. Rev. Lett. 103, 165004 (2009).

[30] P. Manz, G. S. Xu, B. N. Wan, H. Q. Wang, H. Y. Guo, I. Cziegler, N. Fedorczak, C. Holland, S. H. Müller, S. C. Thakur, M. Xu, K. Miki, P. H. Diamond, and G. R. Tynan, Phys. Plasmas 19, 072311 (2012).

[31] G. R. Tynan, M. Xu, P. H. Diamond, J. A. Boedo, I. Cziegler, N. Fedorczak, P. Manz, K. Miki, S. Thakur, L. Schmitz, L. Zeng, E. J. Doyle, G. M. McKee, Z. Yan, G. S. Xu, H. Q. Wang, H. Y. Guo, J. Dong, K. Zhao, J. Cheng, W. Y. Hong, and L. W. Yan, Nucl. Fusion 53, 073053 (2013).

[32] I. Shesterikov, Y. Xu, G. R. Tynan, P. H. Diamond, S. Jachmich, P. Dumortier, M. Vergote, M. Van Schnoor, G. Van Oost, and the TEXTOR Team, Phys. Rev. Lett. 111, 055006 (2013).

[33] Z. Yan, G. R. McKee, R. Fonck, P. Gohil, R. J. Groebner, and T. H. Osborne, Phys. Rev. Lett. 112, 125002 (2014).

[34] I. Cziegler, G. R. Tynan, P. H. Diamond, A. E. Hubbard, J. W. Hughes, J. Irby, and J. L. Terry, Plasma Phys. \& Controlled Fusion 56, 075013 (2014).

[35] I. Cziegler, G. R. Tynan, P. H. Diamond, A. E. Hubbard, J. W. Hughes, J. Irby, and J. L. Terry, Nucl. Fusion 55, 083007 (2015).

[36] T. Kobayashi, K. Itoh, T. Ido, K. Kamiya, S.-I. Itoh, Y. Miura, Y. Nagashima, A. Fujisawa, S. Inagaki, K. Ida, and K. Hoshino,Phys. Rev. Lett. 111, 035002 (2013).

[37] A. Diallo, S. Banerjee, S. J. Zweben, and T. StoltzfusDueck, Nucl. Fusion 57, 066050 (2017).

[38] R. Wilcox, J. Talmadge, D. Anderson, F. Anderson, and J. Lore, Nucl. Fusion 56, 036002 (2016). 